\title{
Adaptive projective lag synchronization of uncertain complex dynamical networks with delay coupling
}

\author{
Ghada Al-mahbashi ${ }^{1 *}$, Mohd Salmi Md Noorani ${ }^{1}$, Sakhinah Abu Bakar ${ }^{1,2}$ and \\ Mohammed Mossa Al-Sawalha²
}

${ }^{*}$ Correspondence:

mahbashighada@yahoo.com

'school of Mathematical Sciences,

Faculty of Science and Technology,

Universiti Kebangsaan Malaysia,

UKM, Bangi, Selangor, Malaysia

Full list of author information is

available at the end of the article

\section{Springer}

\begin{abstract}
This paper investigates the problem of projective lag synchronization behavior with delayed coupling in drive-response dynamical networks model with identical and non-identical nodes. An adaptive control method is designed to achieve the projective lag synchronization with constant time delay and with time-varying coupling delay. In addition the model harbors fully unknown parameters and disturbances. By using Lyapunov stability theory and adaptive laws, the unknown parameters are estimated. In addition, the unknown bounded mismatch and disturbance terms are also overcome by the proposed control. Finally, the simulation results reveal that the states of the dynamical network with delayed coupling can be asymptotically synchronized onto a desired scaling factor under the designed controller. Additionally, the results prove the validity of the proposed method.
\end{abstract}

Keywords: drive-response dynamical networks; projective lag synchronization; adaptive control; disturbance; coupling delay

\section{Introduction}

In the past few years, synchronization of dynamical systems has shown interesting behaviors which have received increasing attention in various fields of industry and various sciences [1-3]. Meanwhile, many kinds of synchronization have been proposed [4-13] and various control methods have been reported to achieve the different kinds of synchronization for complex networks [14-20].

In many practical situations, time delay may cause undesirable dynamic behaviors such as oscillation, instability, and poor performance. Therefore, the development of synchronization of complex dynamical networks with time delays is very important.

In [21] Guo studied lag synchronization of complex networks with non-delay coupling by proposing pinning control. On the basis of adaptive control, Ji et al. [22] proposed a method with lag synchronization between uncertain complex dynamical networks CDNs with constant delay coupling. Wang et al. [23] proposed function projective synchronization (FPS)in CDNs having constant delay coupling and non-identical reference nodes and both network nodes and reference have unknown parameters and bounded external disturbances. Zhang and Zhao [24] investigated both projective and lag synchronization be-

(c) $2015 \mathrm{Al}$-mahbashi et al. This article is distributed under the terms of the Creative Commons Attribution 4.0 International License (http://creativecommons.org/licenses/by/4.0/), which permits unrestricted use, distribution, and reproduction in any medium, provided you give appropriate credit to the original author(s) and the source, provide a link to the Creative Commons license, and indicate if changes were made. 
tween general complex networks via impulsive control. Based on an adaptive feedback controller, projective lag synchronization of the general complex dynamical networks was proposed with non-delay coupling and different nodes [25]. In [26] Rui-Jin et al. proposed several nonlinear controllers to realize the problem of projective synchronization with non-delayed and constant delayed coupling in drive-response dynamical networks consisting of identical nodes and different nodes.

Motivated by the above discussion, the aim of this paper is to deal with the problem of a projective lag synchronization (PLS) scheme in drive-response dynamical networks (DRDNs) model with coupling delayed consisting of identical and different nodes. Both the drive and the network nodes have uncertain parameters and disturbance. Based on Lyapunov stability theory, an adaptive control method is designed to achieve the projective lag synchronization in DRDNs with constant and time-varying coupling delay. Adopting adaptive gains laws, the unknown parameters are estimated. In addition, the controller is designed to overcome the unknown bounded disturbance. In conclusion, the network is asymptotically synchronized with the proposed method. Moreover, numerical simulations are performed to verify the effectiveness of the theoretical results.

The rest of this paper is organized as follows: the DRDNs model with delay coupling is introduced in Section 2. A general method of PLS in a drive-response dynamical networks (DRDNs) model with constant coupling delayed by an adaptive control method is discussed in Section 3. Section 4 deals with a further investigation of PLS in a driveresponse dynamical networks (DRDNs) model with time-varying coupling delayed by using the proposed method. Examples and their simulations are shown in Section 5. Finally, the conclusions are presented in Section 6.

\section{Model description}

Consider a controlled complex dynamical network with delay coupling consisting of $N$ linearly and diffusively different nodes with both uncertain parameters and disturbance, described as follows:

$$
\dot{x}_{i}^{r}(t)=g_{i}\left(x_{i}^{r}(t)\right)+G_{i}\left(x_{i}^{r}(t)\right) \theta_{i}+c \sum_{j=1}^{N} a_{i j} \Gamma x_{j}^{r}\left(t-d_{i}\right)+\Delta_{i}(t)+u_{i}(t), \quad i=1,2, \ldots, N
$$

where $x_{i}^{r}=\left(x_{i 1}^{r}, x_{i 2}^{r}, \ldots, x_{i n}^{r}\right)^{T} \in \mathbf{R}^{n}$ denotes the state vector of the $i$ th node, $g_{i}: \mathbf{R}^{n} \longrightarrow \mathbf{R}^{n}$ and $G_{i}: \mathbf{R}^{n} \longrightarrow \mathbf{R}^{n \times m_{i}}$ are the known continuous nonlinear function matrices determining the dynamic behavior of the node, $\theta_{i}$ is the unknown constant parameter vector, $u_{i} \in \mathbf{R}^{n}$ is the control input, $c$ is the coupling strength, and $d_{i} \geq 0$ is an unknown coupling delay. Here $\Gamma=\operatorname{diag}\left(\gamma_{1}, \gamma_{2}, \ldots, \gamma_{n}\right)$ is the inner coupling matrix with $\gamma_{i}=1$ for the $i$ th state variable, i.e. matrix $\Gamma$ determines the variables with which the nodes in system are coupled. $A=\left(a_{i j}\right)_{N \times N} \in \mathbf{R}^{N \times N}$ is the coupling configuration matrix representing the topological structure of the networks, where $a_{i j}$ is defined as follows: if there exists a connection between node $i$ and node $j(j \neq i)$, then $a_{i j}>0$, otherwise $a_{i j}=0$, and the diagonal elements of matrix $A$ are defined by

$$
a_{i i}=-\sum_{j=1, j \neq i}^{N} a_{i j}, \quad i=1,2, \ldots, N
$$


The reference node is described as follows:

$$
\dot{x}^{d}(t)=f\left(x^{d}(t)\right)+F\left(x^{d}(t)\right) \Phi+\Delta_{d}(t)
$$

where the superscripts $d$ stand for the drive system; $x^{d}=\left(x_{1}^{d}, x_{2}^{d}, \ldots, x_{n}^{d}\right)^{T} \in \mathbf{R}^{n}$ denotes the state vector of the drive system, $f: \mathbf{R}^{n} \longrightarrow \mathbf{R}^{n}$ and $F_{i}: \mathbf{R}^{n} \longrightarrow \mathbf{R}^{n \times m_{i}}$ are the known continuous nonlinear function matrices determining the dynamic behavior of the node; $\Phi$ is the unknown constant parameter vector, and $\Delta_{d}$ contains the mismatched terms.

The projective lag synchronization error is defined as

$$
e_{i}(t)=x_{i}^{r}(t)-\alpha x^{d}(t-\tau), \quad i=1, \ldots, N,
$$

where $\alpha$ is the nonzero a scaling factor, $\tau>0$ is a constant representing time delay or lag. Then the objective of this paper is to design a controller $u_{i}(t)$ such that the reference nodes (1) and dynamical networks (3) are asymptotically synchronized such that

$$
\lim _{t \rightarrow \infty}\left\|x_{i}^{r}(t)-\alpha x^{d}(t-\tau)\right\|=0, \quad i=1, \ldots, N,
$$

which means that the network (1) is projective lag synchronized with reference node (3).

The error dynamics is obtained:

$$
\begin{aligned}
\dot{e}_{i}= & g_{i}\left(x_{i}^{r}(t)\right)+G_{i}\left(x_{i}^{r}(t)\right) \theta_{i}(t)+c \sum_{j=1}^{N} a_{i j} \Gamma e_{j}\left(t-d_{i}\right)+u_{i}(t)+\Delta_{i}(t) \\
& -\alpha\left(f\left(x^{d}(t-\tau)\right)+F\left(x^{d}(t-\tau)\right) \Phi(t)+\Delta_{d}(t)\right), \quad i=1, \ldots, N .
\end{aligned}
$$

Assumption 2.1 [22] For any positive constant $\varepsilon_{i}$ the time-varying disturbance $\Delta_{i}(t)$ is bounded i.e. $\left\|\Delta_{i}(t)\right\| \leq \varepsilon_{i}$.

\section{PLS in DRDNs with constant delay}

In this section, we design an adaptive control method to realize projective lag synchronization for uncertain complex dynamical networks with constant delay coupling.

Theorem 3.1 The projective lag synchronization error (6) is asymptotically stable with a given time delay $\tau$ and scaling factor $\alpha$, by using the following control input and adaptive laws:

$$
\begin{aligned}
u_{i}(t)= & -q_{i} e_{i}(t)-\beta_{i} \operatorname{sgn}\left(e_{i}(t)\right)-g_{i}\left(x_{i}^{r}(t)\right)-G_{i}\left(x_{i}^{r}(t)\right) \hat{\theta}_{i}(t) \\
& +\alpha\left(f\left(x^{d}(t-\tau)\right)+F\left(x^{d}(t-\tau)\right) \hat{\Phi}(t)\right), \quad i=1, \ldots, N, \\
\dot{\hat{\theta}}_{i}(t)= & k_{1} G_{i}^{T}\left(x_{i}^{r}(t)\right) e_{i}(t), \\
\dot{\hat{\Phi}}(t)= & -k_{2} F_{i}^{T}\left(x_{i}^{d}(t-\tau)\right) e_{i}(t), \\
\dot{q}_{i}(t)= & k_{3} e_{i}(t)^{T} e_{i}(t), \\
\dot{\beta}_{i}(t)= & k_{4} e_{i}(t)^{T} \operatorname{sgn}\left(e_{i}(t)\right),
\end{aligned}
$$

where $k_{1}, k_{2}, k_{3}$, and $k_{4}$ are positive constants and $\hat{\Phi}(t)$ and $\hat{\theta}_{i}(t)$ are the estimated parameters for the reference node (1) and network (3), respectively. 
Proof Construct the Lyapunov function candidate as follows:

$$
\begin{aligned}
V(t)= & \frac{1}{2} \sum_{i=1}^{N} e_{i}(t)^{T} e_{i}(t)+\frac{1}{2 k_{1}} \sum_{i=1}^{N} \tilde{\theta}_{i}^{T}(t) \tilde{\theta}_{i}(t)+\frac{1}{2 k_{2}} \sum_{i=1}^{N} \tilde{\Phi}_{i}^{T}(t) \tilde{\Phi}_{i}(t) \\
& +\frac{1}{2 k_{3}} \sum_{i=1}^{N} \tilde{q}_{i}^{2}(t)+\frac{1}{2 k_{4}} \sum_{i=1}^{N} \tilde{\beta}_{i}^{2}(t)+\frac{1}{2} \int_{t-d_{i}}^{t} \sum_{i=1}^{N} e_{i}(s)^{T} e_{i}(s) \mathrm{d} s,
\end{aligned}
$$

where $\tilde{\Phi}_{i}(t)=\hat{\Phi}_{i}(t)-\Phi, \tilde{\theta}_{i}(t)=\hat{\theta}_{i}(t)-\theta_{i}, \tilde{q}_{i}(t)=q_{i}(t)-q_{i}^{*}, \tilde{\beta}_{i}(t)=\beta_{i}(t)-\beta_{i}^{*}$, where $q_{i}^{*}$ and $\beta_{i}^{*}$ are positive constants.

The time derivative of $V(t)$ along the error dynamics (6) is

$$
\begin{aligned}
\dot{V}= & \sum_{i=1}^{N}\left[e_{i}^{T}(t) \dot{e}_{i}(t)+\frac{1}{k_{1}} \dot{\hat{\theta}}_{i}^{T}(t) \tilde{\theta}_{i}(t)+\frac{1}{k_{2}} \dot{\hat{\Phi}}_{i}^{T}(t) \tilde{\Phi}_{i}(t)+\frac{1}{k_{3}} \dot{q}_{i} \tilde{q}_{i}(t)+\frac{1}{k_{4}} \dot{\beta}_{i} \tilde{\beta}_{i}(t)\right] \\
& +\frac{1}{2} \sum_{i=1}^{N} e_{i}(t)^{T} e_{i}(t)-\frac{1}{2} \sum_{i=1}^{N} e_{i}\left(t-d_{i}\right)^{T} e_{i}\left(t-d_{i}\right) .
\end{aligned}
$$

By application of the control input (7) to the error dynamics $\dot{e}_{i}(t)$ we have

$$
\begin{aligned}
\dot{V}= & \sum_{i=1}^{N}\left[e_{i}^{T}(t)\left(-q_{i} e_{i}(t)-\beta_{i}(t) \operatorname{sgn}\left(e_{i}(t)\right)-G_{i}\left(x_{i}^{r}(t)\right) \tilde{\theta}_{i}(t)\right)\right] \\
& +\sum_{i=1}^{N}\left[e_{i}^{T}(t)\left(\alpha F\left(x^{d}(t-\tau)\right) \tilde{\Phi}(t)+c \sum_{j=1}^{N} a_{i j} \Gamma e_{j}\left(t-d_{i}\right)+\Delta_{i}(t)-\alpha \Delta_{d}(t)\right)\right] \\
& +\sum_{i=1}^{N}\left[\frac{1}{k_{1}} \dot{\hat{\theta}}_{i}^{T}(t) \tilde{\theta}_{i}(t)+\frac{1}{k_{2}} \dot{\hat{\Phi}}_{i}^{T}(t) \tilde{\Phi}_{i}(t)+\frac{1}{k_{3}} \dot{q}_{i} \tilde{q}_{i}(t)+\frac{1}{k_{4}} \dot{\beta}_{i} \tilde{\beta}_{i}(t)\right] \\
& +\frac{1}{2} \sum_{i=1}^{N} e_{i}(t)^{T} e_{i}(t)-\frac{1}{2} \sum_{i=1}^{N} e_{i}\left(t-d_{i}\right)^{T} e_{i}\left(t-d_{i}\right)
\end{aligned}
$$

From the adaptation laws (8)-(11), $\dot{V}$ is inferred as follows:

$$
\begin{aligned}
\dot{V}= & -\sum_{i=1}^{N} q^{*} e_{i}^{T}(t) e_{i}(t)-\sum_{i=1}^{N} \beta^{*} e_{i}^{T}(t) \operatorname{sgn}\left(e_{i}(t)\right)+\sum_{i=1}^{N} e_{i}^{T}(t)\left(\Delta_{i}(t)-\alpha \Delta_{d}(t)\right) \\
& +c \sum_{i=1}^{N} e_{i}^{T}(t) \sum_{j=1}^{N} a_{i j} \Gamma e_{j}\left(t-d_{i}\right)+\frac{1}{2} \sum_{i=1}^{N} e_{i}(t)^{T} e_{i}(t)-\frac{1}{2} \sum_{i=1}^{N} e_{i}\left(t-d_{i}\right)^{T} e_{i}\left(t-d_{i}\right) .
\end{aligned}
$$

Let $e(t)=\left(e_{1}^{T}(t), e_{2}^{T}(t), \ldots, e_{N}^{T}(t)\right)^{T} \in \mathbf{R}^{n N}, P=(A \otimes \Gamma)$ where $\otimes$ represents the Kronecker product. Then we have

$$
\begin{aligned}
\dot{V}= & c e^{T}(t) \operatorname{Pe}(t-d)-\sum_{i=1}^{N} q^{*} e_{i}^{T}(t) e_{i}(t)-\sum_{i=1}^{N} \beta^{*} e_{i}^{T}(t) \operatorname{sgn}\left(e_{i}(t)\right) \\
& +\sum_{i=1}^{N} e_{i}^{T}(t)\left(\Delta_{i}(t)-\alpha \Delta_{d}(t)\right)+\frac{1}{2} \sum_{i=1}^{N} e_{i}(t)^{T} e_{i}(t)-\frac{1}{2} \sum_{i=1}^{N} e_{i}\left(t-d_{i}\right)^{T} e_{i}\left(t-d_{i}\right) .
\end{aligned}
$$


We use the fact $2 x^{T} y \leq x^{T} S x+y^{T} S^{-1} y$ for any vector $x, y \in \mathbf{R}^{m}$, and a positive definite matrix $S \in \mathbf{R}^{m \times m}$. From the assumption 1, the following inequality is inferred:

$$
\Delta_{i}(t)-\alpha \Delta_{d}(t) \leq\left\|\left(\Delta_{i}(t)-\alpha \Delta_{d}(t)\right)\right\| \leq\left\|\Delta_{i}(t)\right\|+\alpha\left\|\Delta_{d}(t)\right\| \leq \varepsilon_{i}
$$

where $\varepsilon_{i}$ is a positive constant. We have

$$
\begin{aligned}
\dot{V} \leq & \frac{1}{2} c^{2} e^{T}(t) P^{T} P e(t)+\frac{1}{2} e^{T}\left(t-d_{i}\right) e\left(t-d_{i}\right)-q^{*} e^{T}(t) e(t) \\
& +\sum_{i=1}^{N}\left(\varepsilon_{i}-\beta^{*}\right)\left\|e_{i}(t)\right\|+\frac{1}{2} e(t)^{T} e(t)-\frac{1}{2} e\left(t-d_{i}\right)^{T} e\left(t-d_{i}\right) \\
\leq & e^{T}(t)\left(\frac{1}{2} c^{2} P^{T} P-q^{*}+\frac{1}{2} I\right) e(t)+\sum_{i=1}^{N}\left(\varepsilon_{i}-\beta^{*}\right)\left\|e_{i}(t)\right\| \\
\leq & \left(\lambda-q^{*}\right) e(t)^{T} e(t)+\sum_{i=1}^{N}\left(\varepsilon_{i}-\beta^{*}\right)\left\|e_{i}(t)\right\|,
\end{aligned}
$$

where $\lambda=\lambda_{\max }\left(\frac{c^{2} P^{T} P+I}{2}\right)$. Therefore, by taking appropriate $q^{*}$ and $\beta^{*}$ such that

$$
\begin{aligned}
& \left(\lambda-q^{*}\right)<0, \\
& \varepsilon_{i}-\beta^{*}<0, \quad i=1,2, \ldots, N,
\end{aligned}
$$

we obtain

$$
\dot{V} \leq-e(t)^{T} e(t)
$$

Since $V$ is positive definite and $\dot{V}$ is negative definite, the error $e_{i}(t)$ is asymptotically stable in the sense of Lyapunov stability theory and the networks (3) projective lag synchronizes the drive system (1) asymptotically by the control (7) and the update laws (8)-(11). This completes the proof.

\section{PLS in DRDNs with time-varying delayed coupling}

The adaptive control method is designed to realize projective lag synchronization for uncertain complex dynamical networks with time-varying delay coupling.

Theorem 4.1 We assume a given synchronization scaling factor $\alpha$ and propagation delay $\tau(t)$. The projective lag synchronization with time-varying delayed coupling in the driveresponse dynamical networks can be realized if the control input and adaptive lows are chosen as

$$
\begin{aligned}
u_{i}(t)= & -q_{i} e_{i}(t)-\beta_{i} \operatorname{sgn}\left(e_{i}(t)\right)-g_{i}\left(x_{i}^{r}(t)\right)-G_{i}\left(x_{i}^{r}(t)\right) \hat{\theta}_{i}(t) \\
& +\alpha\left(f\left(x^{d}(t-\tau)\right)+F\left(x^{d}(t-\tau)\right) \hat{\Phi}(t)\right), \quad i=1, \ldots, N, \\
\dot{\hat{\theta}}(t)= & k_{1} G_{i}^{T}\left(x_{i}^{r}(t)\right) e_{i}(t), \\
\dot{\hat{\Phi}}(t)= & -k_{2} F_{i}^{T}\left(x_{i}^{d}(t-\tau)\right) e_{i}(t),
\end{aligned}
$$




$$
\begin{aligned}
& \dot{q}_{i}(t)=k_{3} e_{i}(t)^{T} e_{i}(t), \\
& \dot{\beta}_{i}(t)=k_{4} e_{i}(t)^{T} \operatorname{sgn}\left(e_{i}(t)\right),
\end{aligned}
$$

where $k_{1}, k_{2}, k_{3}$, and $k_{4}$ are positive constants and $\hat{\Phi}(t)$ and $\hat{\theta}_{i}(t)$ are the estimated parameters for the reference node(1) and network (3), respectively.

Proof Choose the Lyapunov function candidate as follows:

$$
\begin{aligned}
V(t)= & \frac{1}{2} \sum_{i=1}^{N} e_{i}(t)^{T} e_{i}(t)+\frac{1}{2 k_{1}} \sum_{i=1}^{N} \tilde{\theta}_{i}^{T}(t) \tilde{\theta}_{i}(t)+\frac{1}{2 k_{2}} \sum_{i=1}^{N} \tilde{\Phi}_{i}^{T}(t) \tilde{\Phi}_{i}(t) \\
& +\frac{1}{2 k_{3}} \sum_{i=1}^{N} \tilde{q}_{i}^{2}(t)+\frac{1}{2 k_{4}} \sum_{i=1}^{N} \tilde{\beta}_{i}^{2}(t) \\
& +\frac{1}{2(1-\eta)} \int_{t-\tau_{1}}^{t} \sum_{i=1}^{N} e_{i}(s)^{T} e_{i}(s) \mathrm{d} s .
\end{aligned}
$$

The rest of the proof is similar to Theorem 3.1 According to the Lyapunov stability theory, the error system is asymptotically stable. This completes the proof.

\section{Illustrative example}

This section presents the drive-response dynamical networks with three identical, different nodes systems, unknown parameters, and disturbance, which are used to show the effectiveness of the proposed schemes obtained in the previous sections. We use the Lorenz system as drive system, which is described as follows:

$$
\left(\begin{array}{c}
\dot{x}_{1}^{d} \\
\dot{x}_{2}^{d} \\
\dot{x}_{3}^{d}
\end{array}\right)=\left(\begin{array}{c}
0 \\
-x_{1}^{d} x_{3}^{d}-x_{2}^{d} \\
x_{1}^{d} x_{2}^{d}
\end{array}\right)+\left(\begin{array}{ccc}
x_{2}^{d}-x_{1}^{d} & 0 & 0 \\
0 & x_{1}^{d} & 0 \\
0 & 0 & -x_{3}^{d}
\end{array}\right)\left(\begin{array}{c}
\Phi_{1} \\
\Phi_{2} \\
\Phi_{3}
\end{array}\right)+\Delta_{d}(t),
$$

where the unknown parameters vector and mismatch terms are chosen as $\Phi=$ $\left[\Phi_{1} \Phi_{2} \Phi_{3}\right]=\left[1028 \frac{8}{3}\right], \Delta_{d}(t)=[2 \cos (t) 3 \sin (t) \sin (t)]$.

The inner coupling matrix $\Gamma=I_{3 \times 3}$ and the coupling configuration matrix $A=\left(a_{i j}\right)$ is chosen to be

$$
A=\left[\begin{array}{ccc}
-2 & 1 & 1 \\
1 & -1 & 0 \\
0 & 1 & -1
\end{array}\right]
$$

\subsection{Synchronization with constant delay}

We discuss the problem of PLS in drive-response dynamical networks with identical and different nodes consisting of fully unknown parameters, mismatch terms, and disturbance with constant delay coupling.

\subsubsection{Synchronization with identical nodes}

We take a chaotic Chen system as the $i$ th networks nodes with unknown parameters and disturbance to realize PLS in DRDNs and verify the effectiveness of the proposed scheme 

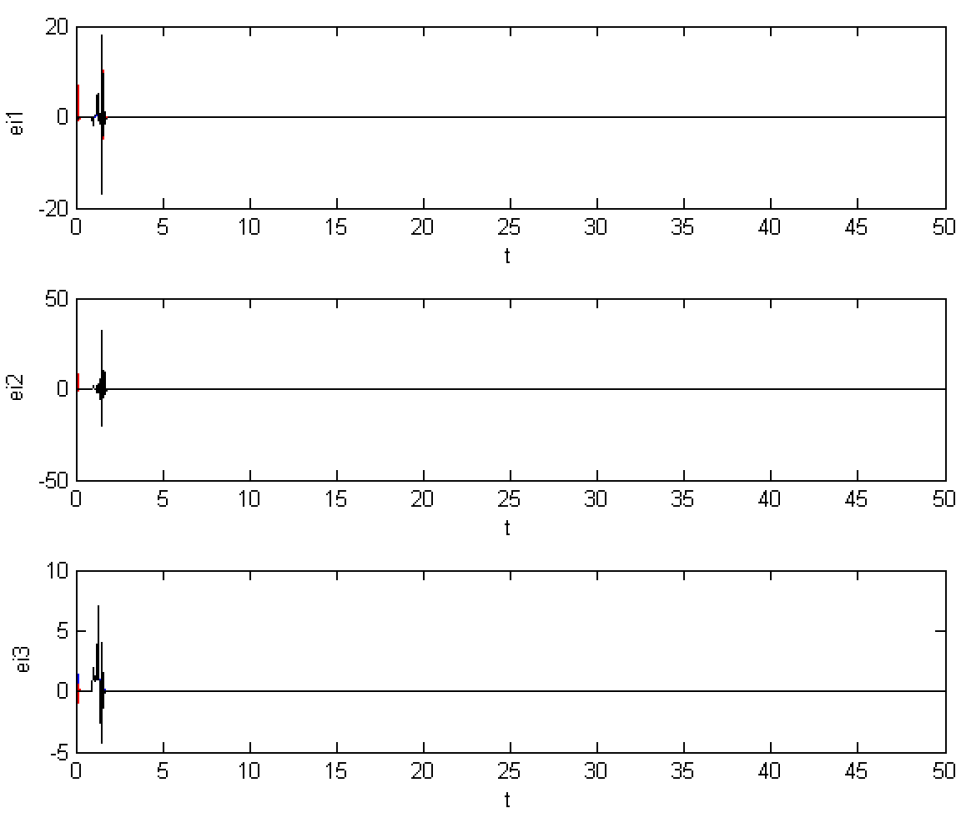

Figure 1 The synchronization error $e_{i}(t)=x_{i}^{r}(t)-\alpha x^{d}(t-\tau)$.

which can be described as follows:

$$
\begin{aligned}
\left(\begin{array}{c}
\dot{x}_{i 1}^{r} \\
\dot{x}_{i 2}^{r} \\
\dot{x}_{i 3}^{r}
\end{array}\right)= & \left(\begin{array}{c}
0 \\
-x_{i 1}^{r} x_{i 3}^{r} \\
x_{i 1}^{r} x_{i 2}^{r}
\end{array}\right)+\left(\begin{array}{ccc}
x_{i 2}^{r}-x_{i 1}^{r} & 0 & 0 \\
-x_{i 1}^{r} & x_{i 1}^{r}+x_{i 2}^{r} & 0 \\
0 & 0 & -x_{i 3}^{r}
\end{array}\right)\left(\begin{array}{l}
\theta_{i 1} \\
\theta_{i 2} \\
\theta_{i 3}
\end{array}\right) \\
& +c \sum_{j=1}^{3} a_{i j} \Gamma x_{j}^{r}\left(t-d_{i}\right)+\Delta_{i}(t)+u_{i} .
\end{aligned}
$$

Here the unknown parameters vector is $\theta_{1}=\left[\begin{array}{lll}35 & 28 & 3\end{array}\right]^{T}$. The disturbance signals are chosen as $\Delta_{i}=[0.3 \cos (t) \sin (t) 0.1 \sin (t) 0.5 \cos (t)]$.

In these numerical simulations, we assume that $c=0.2, \alpha=2, d_{i}=0.2$, and $\tau=1$. The gains of the adaptive laws (8)-(11) are $k_{1}=9, k_{2}=8, k_{3}=1, k_{4}=0.8$. We take the initial states as $x^{d}(0)=[1-1-1]^{T}, x_{i}^{r}(0)$ are chosen in $[-5,5]$ randomly, and $\hat{\Phi}_{0}=\hat{\theta}_{i 0}=q_{i 0}=\beta_{i 0}=0$.

The numerical results are presented in Figures 1 and 2 . The time evolution of the synchronization errors is illustrated in Figure 1, which displays $e \longrightarrow 0$ with $t \longrightarrow \infty$. The identified parameters of the reference node and network nodes are depicted in Figure 2(a) and Figure 2(b), which converge to their real values. These results verify that the proposed control (7) with adaptive laws (8)-(11) makes the network (3) projective lag synchronized, even if the network and the reference node (1) have fully unknown parameters, mismatch terms, and disturbances.

\subsubsection{Synchronization with different nodes}

The Chen system, the Lu system, and the Rossler system as the response networks with constant delayed coupling, respectively, are described as follows: 

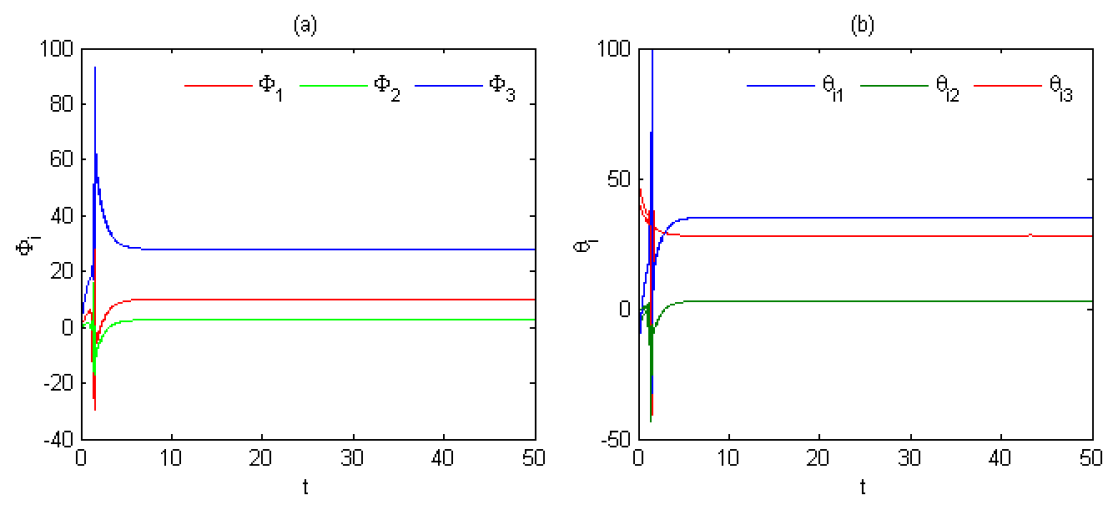

Figure 2 The estimated parameters $\hat{\phi}_{i}(\mathrm{a})$ and $\hat{\theta}_{i}(\mathrm{~b})$.

$$
\begin{aligned}
\left(\begin{array}{l}
\dot{x}_{11}^{r} \\
\dot{x}_{12}^{r} \\
\dot{x}_{13}^{r}
\end{array}\right)= & \left(\begin{array}{c}
0 \\
-x_{11}^{r} x_{13}^{r} \\
x_{11}^{r} x_{12}^{r}
\end{array}\right)+\left(\begin{array}{ccc}
x_{12}^{r}-x_{11}^{r} & 0 & 0 \\
-x_{11}^{r} & x_{11}^{r}+x_{12}^{r} & 0 \\
0 & 0 & -x_{13}^{r}
\end{array}\right)\left(\begin{array}{l}
\theta_{11} \\
\theta_{12} \\
\theta_{13}
\end{array}\right) \\
& +c \sum_{j=1}^{3} a_{1 j} \Gamma x_{j}^{r}\left(t-d_{1}\right)+u_{1}(t), \\
\left(\begin{array}{l}
\dot{x}_{21}^{r} \\
\dot{x}_{22}^{r} \\
\dot{x}_{23}^{r}
\end{array}\right)= & \left(\begin{array}{c}
0 \\
-x_{21}^{r} x_{23}^{r} \\
x_{21}^{r} x_{22}^{r}
\end{array}\right)+\left(\begin{array}{ccc}
x_{22}^{r}-x_{21}^{r} & 0 & 0 \\
0 & x_{22}^{r} & 0 \\
0 & 0 & -x_{23}^{r}
\end{array}\right)\left(\begin{array}{l}
\theta_{21} \\
\theta_{22} \\
\theta_{23}
\end{array}\right) \\
& +c \sum_{j=1}^{3} a_{2 j} \Gamma x_{j}^{r}\left(t-d_{2}\right)+u_{2}(t), \\
\left(\begin{array}{l}
\dot{x}_{31}^{r} \\
\dot{x}_{32}^{r} \\
\dot{x}_{33}^{r}
\end{array}\right)= & \left(\begin{array}{l}
-x_{32}^{r}-x_{33}^{r} \\
x_{31}^{r} \\
x_{31}^{r} x_{32}^{r}+0.2
\end{array}\right)+\left(\begin{array}{cc}
0 & 0 \\
x_{32}^{r} & 0 \\
0 & -x_{33}^{r}
\end{array}\right)\left(\begin{array}{l}
\theta_{31} \\
\theta_{32}
\end{array}\right) \\
& +c \sum_{j=1}^{3} a_{3 j} \Gamma x_{j}^{r}\left(t-d_{3}\right)+u_{3}(t) .
\end{aligned}
$$

In these numerical simulations, we assume that $c=0.2, \alpha=2, d_{i}=0.2$, and $\tau=1$. The gains of the adaptive laws (8)-(11) are $k_{1}=9, k_{2}=9, k_{3}=1, k_{4}=2$, and $q_{i}=0$. We take the initial states as $x^{d}(0)=\left[\begin{array}{lll}1 & 2 & 3\end{array}\right]^{T}, x_{i}^{r}(0)$ are chosen in $[-5,5]$ randomly, and $\hat{\Phi}_{0}=\hat{\theta}_{i 0}=q_{i 0}=$ $\beta_{i 0}=0$.

The time evolution of the synchronization errors is illustrated in Figure 3, which displays $e \longrightarrow 0$ with $t \longrightarrow \infty$. The estimated parameters of the reference node and network nodes are depicted in Figure 4(a) and Figure 4(b), respectively, which converge to their real values. These results prove that the proposed control (7) with adaptive laws (8)-(10) makes the network (3) projective lag synchronized if the drive system and the network have unknown parameters. 

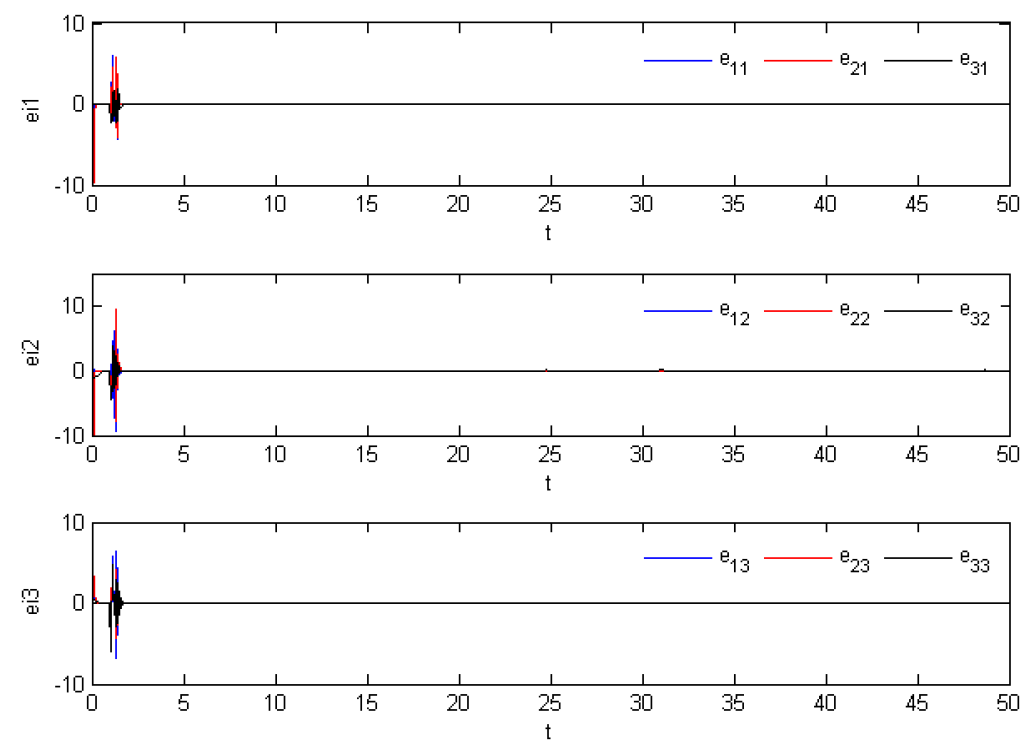

Figure 3 The synchronization error of different nodes.
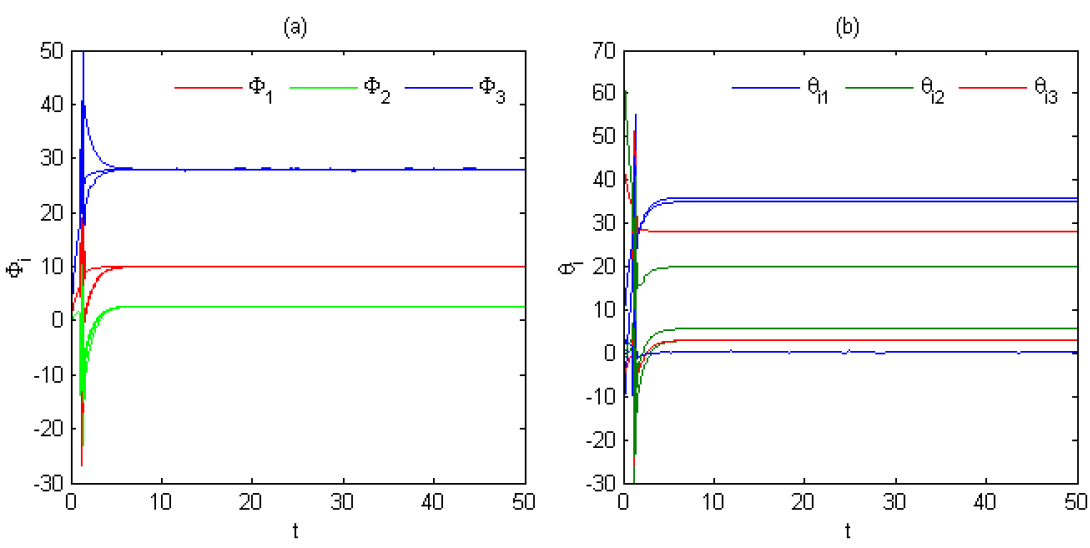

Figure 4 The estimated parameters $\hat{\phi}_{i}(\mathrm{a})$ and $\hat{\theta}_{i}(\mathrm{~b})$ with constant coupling delay.

\subsection{Synchronization with varying coupling delay coupling}

In this subsection, a drive-response dynamical networks with three identical, different node systems, fully unknown parameters, mismatch, and disturbance terms are used to show the effectiveness of the proposed schemes obtained in the previous sections.

\subsubsection{Synchronization with identical nodes}

The chaotic Chen system is chosen as three nodes of complex dynamical networks; the complex dynamical networks with time-varying coupling delay can be described as follows: 

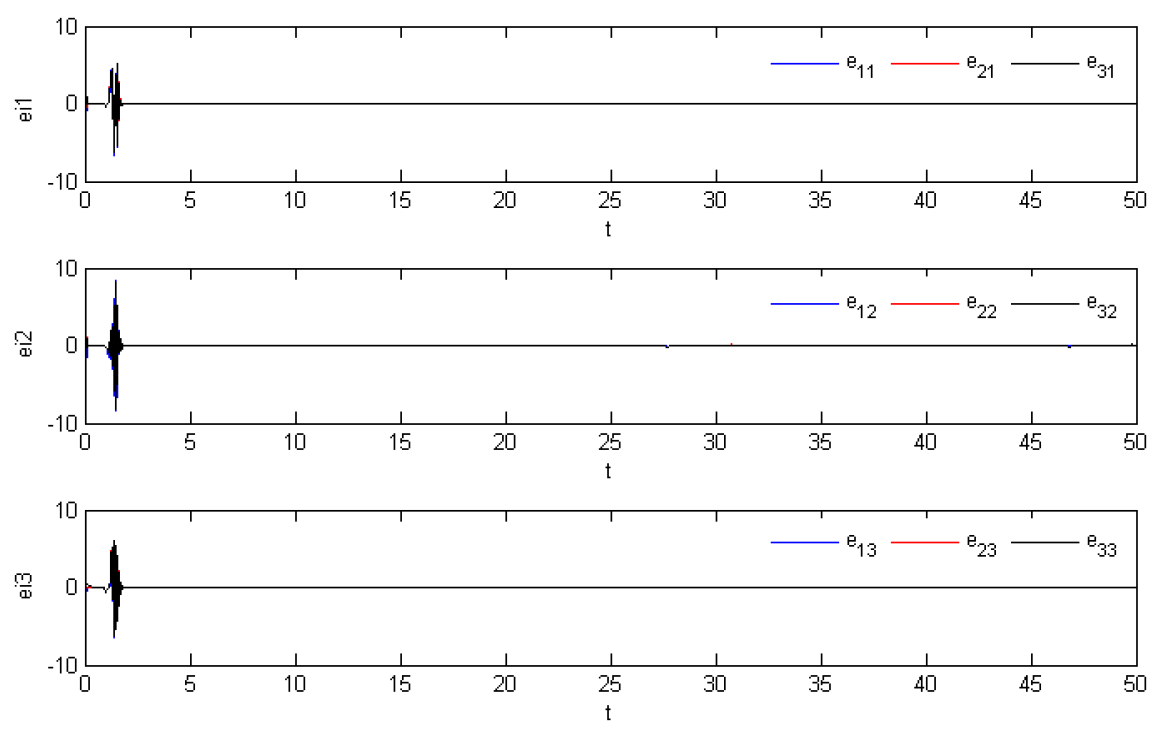

Figure 5 The synchronization error of identical nodes with time-varying delay.

$$
\begin{aligned}
\left(\begin{array}{c}
\dot{x}_{i 1}^{r} \\
\dot{x}_{i 2}^{r} \\
\dot{x}_{i 3}^{r}
\end{array}\right)= & \left(\begin{array}{c}
0 \\
-x_{i 1}^{r} x_{i 3}^{r} \\
x_{i 1}^{r} x_{i 2}^{r}
\end{array}\right)+\left(\begin{array}{ccc}
x_{i 2}^{r}-x_{i 1}^{r} & 0 & 0 \\
-x_{i 1}^{r} & x_{i 1}^{r}+x_{i 2}^{r} & 0 \\
0 & 0 & -x_{i 3}^{r}
\end{array}\right)\left(\begin{array}{l}
\theta_{i 1} \\
\theta_{i 2} \\
\theta_{i 3}
\end{array}\right) \\
& +c \sum_{j=1}^{3} a_{i j} \Gamma x_{j}^{r}\left(t-\tilde{d}_{i}(t)\right)+\Delta_{i}(t)+u_{i} .
\end{aligned}
$$

The propagation delay $d_{i}(t)=1+0.2 \sin (t)$ and $\tau=1$. The gains of the adaptive laws (17)(20) are $k_{1}=15, k_{2}=10, k_{3}=1, k_{4}=0.9$. We take the initial states as $x^{d}(0)=\left[\begin{array}{lll}0.2 & 0.1 & 0.3\end{array}\right]^{T}$, $x_{i}^{r}(0)$ are chosen in $[-5,5]$ randomly, and $\hat{\Phi}_{0}=\hat{\theta}_{i 0}=q_{i 0}=\beta_{i 0}=0$.

The time evolution of the synchronization errors is depicted in Figure 5, which displays $e \longrightarrow 0$ with $t \longrightarrow \infty$. The estimated parameters of the reference node and network nodes are depicted in Figure 6(a) and Figure 6(b), which converge to their real values. These results verify that the proposed control (21) with adaptive laws (17)-(20) makes the network (3) projective lag synchronized.

\subsubsection{Synchronization with different nodes}

The chaotic Chen system, the Lu system, and the Rossler system are chosen as nodes of complex dynamical networks; complex dynamical networks with time-varying coupling delay can be described as follows:

$$
\begin{aligned}
\left(\begin{array}{l}
\dot{x}_{11}^{r} \\
\dot{x}_{12}^{r} \\
\dot{x}_{13}^{r}
\end{array}\right)= & \left(\begin{array}{c}
0 \\
-x_{11}^{r} x_{13}^{r} \\
x_{11}^{r} x_{12}^{r}
\end{array}\right)+\left(\begin{array}{ccc}
x_{12}^{r}-x_{11}^{r} & 0 & 0 \\
-x_{11}^{r} & x_{11}^{r}+x_{12}^{r} & 0 \\
0 & 0 & -x_{13}^{r}
\end{array}\right)\left(\begin{array}{l}
\theta_{11} \\
\theta_{12} \\
\theta_{13}
\end{array}\right) \\
& +c \sum_{j=1}^{3} a_{1 j} \Gamma x_{j}^{r}\left(t-\tilde{d}_{1}(t)\right)+u_{1}(t),
\end{aligned}
$$


(a)

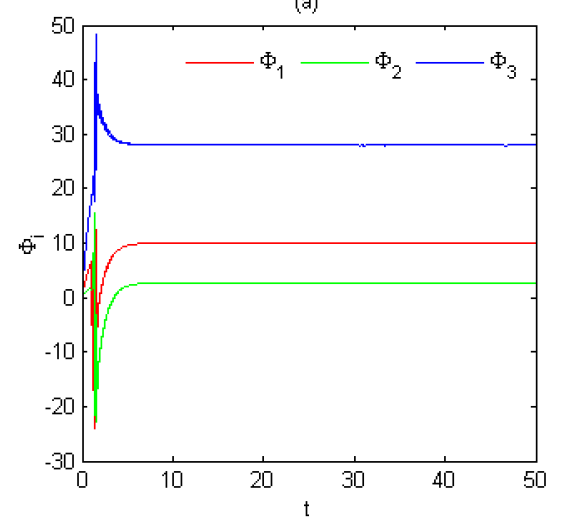

(b)

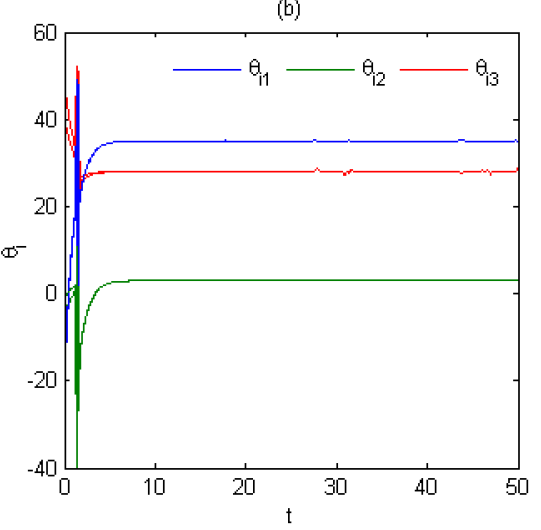

Figure 6 The estimated parameters $\hat{\phi}_{i}(\mathrm{a})$ and $\hat{\theta}_{i}(\mathrm{~b})$ with time-varying coupling delay.

$$
\begin{aligned}
\left(\begin{array}{l}
\dot{x}_{21}^{r} \\
\dot{x}_{22}^{r} \\
\dot{x}_{23}^{r}
\end{array}\right)= & \left(\begin{array}{c}
0 \\
-x_{21}^{r} x_{23}^{r} \\
x_{21}^{r} x_{22}^{r}
\end{array}\right)+\left(\begin{array}{ccc}
x_{22}^{r}-x_{21}^{r} & 0 & 0 \\
0 & x_{22}^{r} & 0 \\
0 & 0 & -x_{23}^{r}
\end{array}\right)\left(\begin{array}{l}
\theta_{21} \\
\theta_{22} \\
\theta_{23}
\end{array}\right) \\
& +c \sum_{j=1}^{3} a_{2 j} \Gamma x_{j}^{r}\left(t-\tilde{d}_{2}(t)\right)+u_{2}(t), \\
\left(\begin{array}{l}
\dot{x}_{31}^{r} \\
\dot{x}_{32}^{r} \\
\dot{x}_{33}^{r}
\end{array}\right)= & \left(\begin{array}{c}
-x_{32}^{r}-x_{33}^{r} \\
x_{31}^{r} \\
x_{31}^{r} x_{32}^{r}+0.2
\end{array}\right)+\left(\begin{array}{cc}
0 & 0 \\
x_{32}^{r} & 0 \\
0 & -x_{33}^{r}
\end{array}\right)\left(\begin{array}{l}
\theta_{31} \\
\theta_{32}
\end{array}\right) \\
& +c \sum_{j=1}^{3} a_{3 j} \Gamma x_{j}^{r}\left(t-\tilde{d}_{3}(t)\right)+u_{3}(t) .
\end{aligned}
$$

In these numerical simulations, we assume the time delay $d_{i}(t)=1+0.2 \sin (t)$ and $\tau=1$. The gain of the adaptive laws (17)-(20) are $k_{1}=7, k_{2}=9, k_{3}=1, k_{4}=2.5$. We take the initial

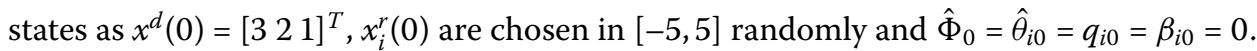

In Figure 7 shows the time evolution of the synchronization errors. The estimated parameters of the reference node and network nodes are depicted in Figure 8(a) and Figure 8 (b), respectively, which converge to their real values. These results verify that the proposed control (21) with adaptive laws (17)-(19) makes the network (3) projective lag synchronized, even though the drive system and the network have unknown parameters.

\section{Conclusion}

An adaptive projective lag synchronization (PLS) scheme was proposed in drive-response dynamical networks with delayed coupling consisting of identical and different nodes. Both of the reference node and network nodes have fully unknown parameters and disturbances. Adaptive control and update laws were designed to achieve the PLS with constant time delay and with time-varying coupling delay. Based on the Lyapunov stability theory and adaptive laws the unknown parameters were estimated. Furthermore, the unknown bounded disturbances were also overcome by the proposed control. The numerical results showed the effectiveness of the proposed approach. 

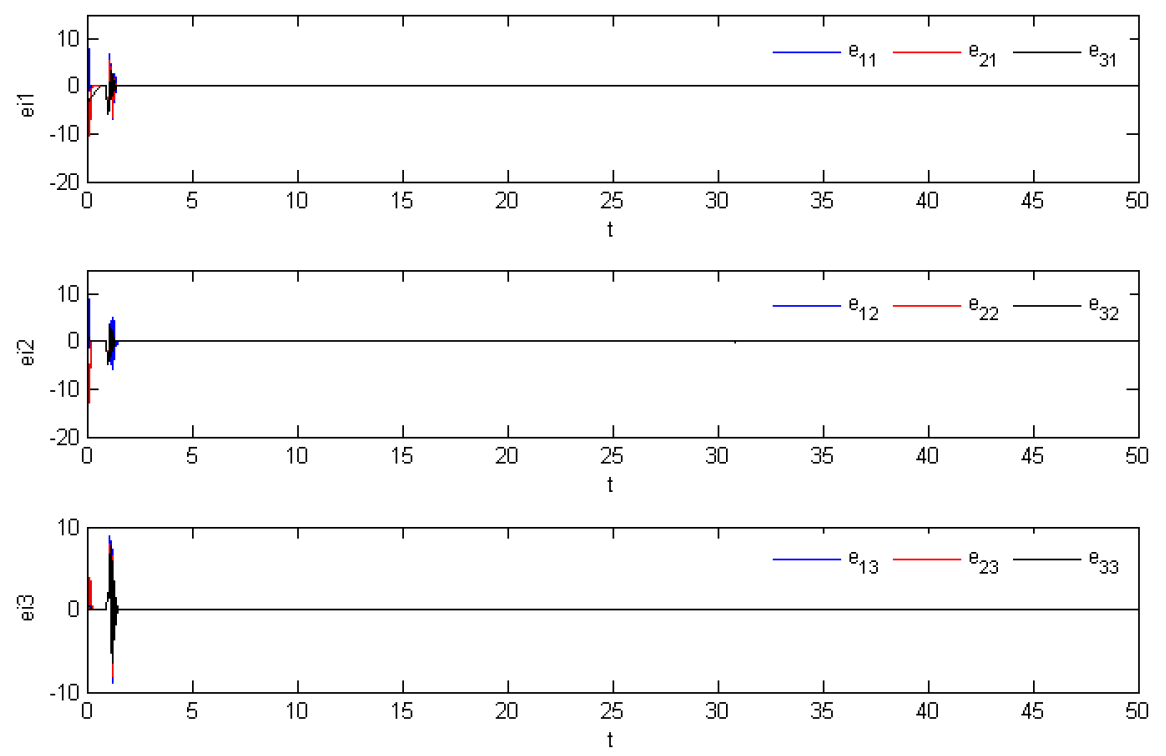

Figure 7 The synchronization error of different nodes with time-varying delay.
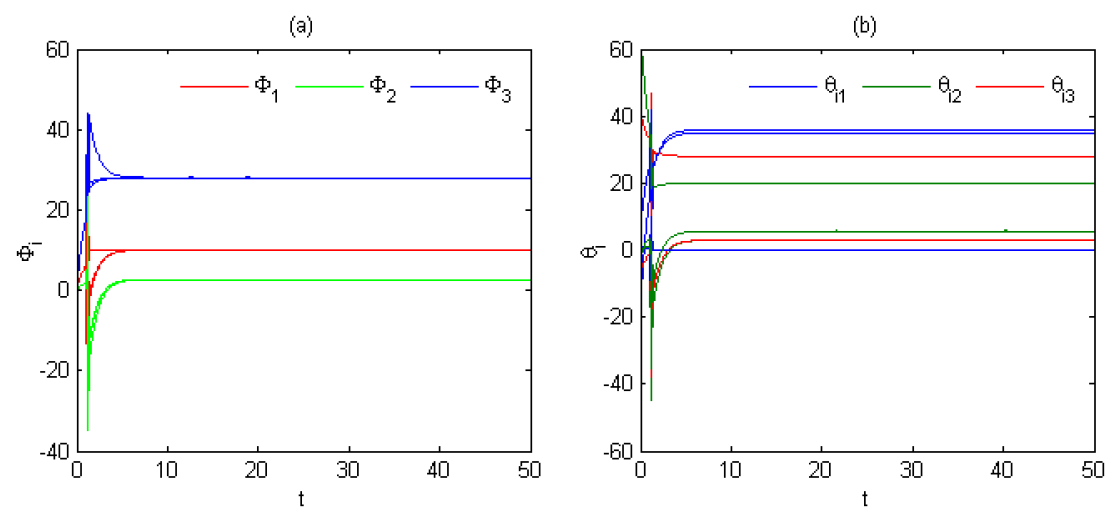

Figure 8 The estimated parameters $\hat{\phi}_{i}$ (a) and $\hat{\theta}_{i}(\mathrm{~b})$ with time-varying coupling delay.

\section{Competing interests}

The authors declare that they have no competing interests.

\section{Authors' contributions}

All authors contributed equally to this work. They all read and approved the final version of the manuscript.

\section{Author details}

'School of Mathematical Sciences, Faculty of Science and Technology, Universiti Kebangsaan Malaysia, UKM, Bangi, Selangor, Malaysia. ${ }^{2}$ Department of Mathematics, Faculty of Science, University of Hail, Hail, Saudi Arabia.

\section{Acknowledgements}

The authors would like to acknowledge the grant: UKM Grant DIP-2014-034 and Ministry of Education, Malaysia grant FRGS/1/2014/ST06/UKM/01/1 for financial support.

Received: 30 June 2015 Accepted: 10 November 2015 Published online: 18 November 2015

\section{References}

1. Pandit, SA, Amritkar, RE: Characterization and control of small-world networks. Phys. Rev. E 60(2), 1119 (1999)

2. Strogatz, SH: Exploring complex networks. Nature 410, 268-276 (2001)

3. Liu, X, Chen, TP: Synchronization analysis for nonlinearly-coupled complex networks with an asymmetrical coupling matrix. Physica A 387(16), 4429-4439 (2008) 
4. Xiao, Y, Xu, W, Li, X, Tang, S: Adaptive complete synchronization of chaotic dynamical network with unknown and mismatched parameters. Chaos 17, 033118 (2007)

5. Shahverdiev, EM, Sivaprakasam, S, Shore, KA: Lag synchronization in time-delayed systems. Phys. Lett. A 292, 320-324 (2002)

6. $\mathrm{Wu}, \mathrm{Y}, \mathrm{Li}, \mathrm{C}, \mathrm{Wu}, \mathrm{Y}, \mathrm{Kurths}, \mathrm{J}$ : Generalized synchronization between two different complex networks. Commun. Nonlinear Sci. Numer. Simul. 17(1), 349-355 (2012). doi:10.1016/j.cnsns.2011.04.026

7. Length, F: Anti-synchronization of complex delayed dynamical networks through feedback control. Sci. Res. Essays 6(3), 552-558 (2011). doi:10.5897/SRE10.760

8. Liu-Xiao, G, Zhen-Yuan, X, Man-Ferg, H: Adaptive projective synchronization with different scaling factors in networks. Chin. Phys. B 17(11), 4067 (2008)

9. Liu, J, Liu, S, Yuan, C: Modified generalized projective synchronization of fractional-order chaotic Lu systems. Adv. Differ. Equ. 2013, 374 (2013)

10. Botmart, T, Niamsup, P: Exponential synchronization of complex dynamical network with mixed time-varying and hybrid coupling delays via intermittent control. Adv. Differ. Equ. 2014, 116 (2014)

11. Zhang, S, Yu, Y, Wen, G, Rahmani, A: Stochastic quasi-synchronization for uncertain chaotic delayed neural networks. Int. J. Mod. Phys. C 25, 1450029 (2014)

12. Wang, S, Cao, H: Cluster lag synchronization of complex networks with nonidentical dynamical nodes via adaptive control. In: 2015 International Conference on Automation, Mechanical Control and Computational Engineering. Atlantis Press (2015)

13. Louzada, VHP, Araujo, NAM, Andrade, JS, Herrmann, HJ: Breathing synchronization in interconnected networks. Sci. Rep. 3, 3289 (2013)

14. $\mathrm{Wu}, \mathrm{Y}, \mathrm{Liu}, \mathrm{L}:$ Exponential outer synchronization between two uncertain time-varying complex networks with nonlinear coupling. Entropy 17(5), 3097-3109 (2015)

15. Al-Mahbashi, G, Noorani, MS, Abu Bakar, S: Projective lag synchronization in drive-response dynamical networks. Int. J. Mod. Phys. C (2014). doi:10.1142/S0129183114500685

16. Wen, B, Zhao, M, Meng, F: Pinning synchronization of the drive and response dynamical networks with lag. Arch. Control Sci. 24(3), 257-270 (2014)

17. Zhao, $M$, Zhang, $\mathrm{H}$, Wang, Z, Liang, H: Synchronization between two general complex networks with time-delay by adaptive periodically intermittent pinning control. Neurocomputing 144, 215-221 (2014)

18. Zheng, S: Projective synchronization analysis of drive-response coupled dynamical network with multiple time-varying delays via impulsive control. Abstr. Appl. Anal. (2014). doi:10.1155/2014/581971

19. Sun, Y, Li, W, Ruan, J: Generalized outer synchronization between complex dynamical networks with time delay and noise perturbation. Commun. Nonlinear Sci. Numer. Simul. 18(4), 989-998 (2013)

20. Sun, HY, Li, N, Zhao, DP, Zhang, QL: Synchronization of complex networks with coupling delays via adaptive pinning intermittent control. Int. J. Autom. Comput. 10(4), 312-318 (2013)

21. Guo, W: Lag synchronization of complex networks via pinning control. Nonlinear Anal., Real World Appl. 12(5), 2579-2585 (2011). doi:10.1016/j.nonrwa.2011.03.007

22. Ji, DH, Jeong, SC, Park, JH, Lee, SM, Won, SC: Adaptive lag synchronization for uncertain complex dynamical network with delayed coupling. Appl. Math. Comput. 218(9), 4872-4880 (2012). doi:10.1016/j.amc.2011.10.051

23. Wang, L, Yuan, Z, Chen, X, Zhou, Z: Adaptive function projective synchronization of uncertain complex dynamical networks with disturbance. Commun. Nonlinear Sci. Numer. Simul. 16, 987-992 (2011)

24. Zhang, Q, Zhao, J: Projective and lag synchronization between general complex networks via impulsive control. Nonlinear Dyn. 67(4), 2519-2525 (2011). doi:10.1007/s11071-011-0164-6

25. $\mathrm{Wu}, \mathrm{X}, \mathrm{Lu}, \mathrm{H}$ : Projective lag synchronization of the general complex dynamical networks with distinct nodes. Commun. Nonlinear Sci. Numer. Simul. 17(11), 4417-4429 (2012)

26. Rui-Jin, D, Gao-Gao, D, Li-Xin, T, Song, Z, Mei, S: Projective synchronisation with non-delayed and delayed coupling in complex networks consisting of identical nodes and different nodes. Chin. Phys. B 19(7), 070509 (2010)

\section{Submit your manuscript to a SpringerOpen ${ }^{\circ}$ journal and benefit from:}

- Convenient online submission

Rigorous peer review

- Immediate publication on acceptance

- Open access: articles freely available online

- High visibility within the field

- Retaining the copyright to your article 